\title{
Application of Method of Equivalent Replacement Beam for Displacement Computation of Statically Indeterminate Structures
}

\author{
Yanxia, XUE ${ }^{1, a}$, Zhenchao, SU ${ }^{1, b, *}$ \\ ${ }^{1}$ Department of Civil Engineering, Tan Kah Kee College, Xiamen University, 363105, Zhangzhou \\ city, Fujian Province, China \\ axueyx888@126.com, buzc888@126.com \\ ${ }^{*}$ Corresponding author
}

Keywords: statically indeterminate beam, method of equivalent replacement beams, deformation computation, mechanics of materials.

Abstract. Based on the method of equivalent replacement beams(ERB) and superposition method for computing deflections of beams, deformation computation of statically indeterminate beams with the method of ERB is discussed. First, the method of ERB is introduced. Second, the conclusion of deformation computation of statically indeterminate beams with the method of equivalent replacement beams or rigid frames is built. Third, the application of method of ERB for deformation computation of statically indeterminate beams(or rigid frames) is demonstrated by examples. These illustrative examples reveals that this method can be used to solve complicit deformation computation of statically indeterminate beams and rigid frames efficiently.

\section{Introduction}

A beam is a structural element that primarily resists loads applied laterally to the beam's axis. The Bernoulli beam is named after Jacob Bernoulli, who made the significant discoveries. Leonhard Euler and Daniel Bernoulli were the first to put together a useful theory circa $1750^{[1]}$. Euler-Bernoulli beam theory is a simplification of the linear theory of elasticity which provides a means of calculating the load-carrying and deflection characteristics of beams ${ }^{[2]}$. It is well known that the calculation of deflections is an important part of structural analysis and design. For instance, large deflections in buildings are unsightly (and even unnerving) and can cause cracks in ceilings and walls. ${ }^{[3]}$

The deflection of Euler-Bernoulli beams can be calculated by the method of integration of the curvature equation, or by methods such as, superposition method, conjugate beam method, moment-area method, virtual work method, Castigliano's method, Macaulay's method, finite difference method $^{[3,4]}$, initial parameter method ${ }^{[5]}$, etc. The method of ERB ${ }^{[6]}$ proposed by SU and XUE is an effective novel method based on superposition method to calculate the displacements for a broad category of statically determinate beams.

In this paper, we will gain an insight into the application of the method of ERB to investigate the displacements of statically indeterminate beams and rigid frames, and extend the method of the ERB to apply for statically indeterminate beams and rigid frames. The article is organized as follows. In Section 2, a brief introduction to the method of ERB is given. In Section 3, a proposition for solving displacements of statically indeterminate beams and rigid frames is proposed. In Section 4, two illustrative examples of using Method of ERB to calculate deflections and rotations of statically indeterminate beans are presented to demonstrate the Method of ERB's effectiveness for statically indeterminate beams and rigid frames.

\section{Introduction to Method of ERB}

As depicted in [6], an ERB of a beam is a cantilever beam can be regarded as a substitute of another type of beam in the sense of having the equivalent (inner and outer) forces and equivalent deflection at all sections over the total beam in addition to same material and cross section shape. 
The properties of cantilever beams are simple knowledge in many current university textbooks ${ }^{\text {[4-11] }}$ of mechanics of materials. For example, the deflection and angle of rotation of cantilever beam $A B$ acted with a couple $M_{\mathrm{e}}$ at free end $B$ shown in Fig.1- Fig.3 are expressed by (1)-(3) as below.

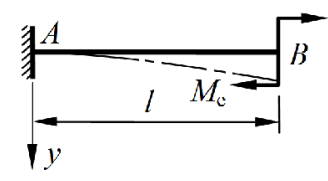

Fig.1 Cantilever beam acted with a couple

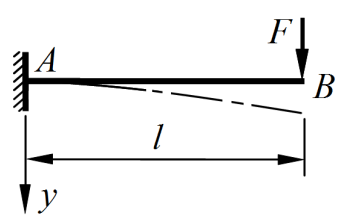

Fig.2 Cantilever beam acted with a concentrated force

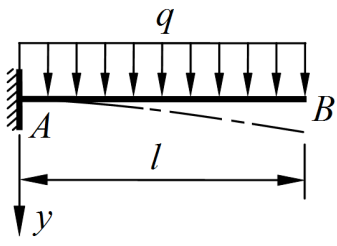

Fig.3 Cantilever beam acted with uniform load

$$
\begin{aligned}
& \theta_{B}=\frac{M_{\mathrm{e}} l}{E I}(\mathrm{P}), \quad w_{B}=\frac{M_{\mathrm{e}} l^{2}}{2 E I}(\$) \\
& \theta_{B}=\frac{F l^{2}}{2 E I}(\mathrm{P}), \quad w_{B}=\frac{F l^{3}}{3 E I}(\$) \\
& \theta_{B}=\frac{q l^{3}}{6 E I}(\mathrm{P}), \quad w_{B}=\frac{q l^{4}}{8 E I}(\$)
\end{aligned}
$$

in which, $w_{B}$ and $\theta_{B}$ are the deflection and angle of rotation of the beam at the end $B, E I$ is the flexural rigidity of the beam and $l$ is its length.

These results are essential and important for engineering practice. At the same time, they play a fundamental role in the course of structural mechanics or engineering structure. In this section, based on the above results, we will try to use cantilever beams to replace other type of beams to analyse the deflections of various types of beams.

\section{Method of ERB for statically indeterminate beams}

First, the conclusion of method of ERB for computing deflections of statically determinate beams is stated as follows ${ }^{[6]}$.

For a straight statically determinate beam with constant section, its ERB must exist as long as the deflection and angle of rotation of ERB at the fixed end equals to its origin, and the actions on ERB are the same as its origin.

Furthermore, for statically indeterminate beams or rigid frames, the corresponding result is hold.

For a statically indeterminate beam (determinate or indeterminate rigid frame) with constant section, it can be replaced by an ERB or a series of ERBs as long as the deflections and the slopes of the ERBs at the fixed ends equal to their origin respectively, and the actions on ERBs are the same as their originals.

\section{Application of method of ERB for statically indeterminate beams or rigid frames}

Example 1. A two-span continuous beam $A B C$ supports a concentrated load $F$, as shown in Fig.4. Determine the angle of rotation $\theta_{B}$ at support $B$, deflection and rotation of sections $D$ using method of ERB.

Solution. Assuming that the unknown reactive forces at $A, B$ and $C$ respectively are $F_{A}, F_{B}$ and $F_{C}$, and the angle of rotation of cross section $B$ is $\theta_{B}$, fixed the cross section $B$ after rotating clock-wisely the beam $A B C$, and applying the forces $F_{A}, F_{B}$ and $F_{C}$ on it, then the ERB of beam $A B C$ in Fig.4 is built with $A_{1} B_{1} C_{1}$ as shown in Fig.5.

By the geometric relations in Fig.5, one reads 
$\overline{A A_{1}}=a \cdot \theta_{B}, \overline{C C_{1}}=(b+c) \cdot \theta_{B}$

Employing the results of (3) and (4), one reads

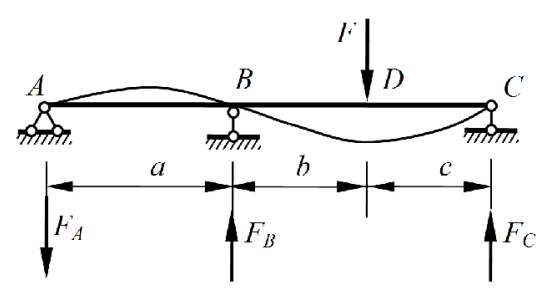

Fig.4 Indeterminate beam with a concentrated load

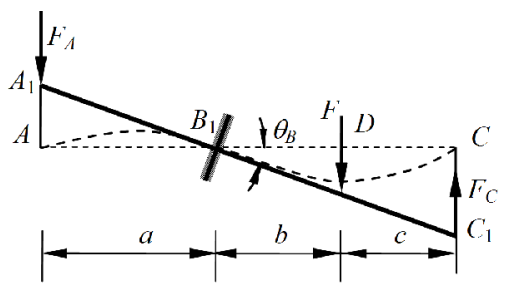

Fig.5 The ERB of beam $A B C$ in Fig.4

$\frac{F_{A} a^{3}}{3 E I}-a \cdot \theta_{B}=0$

$\frac{F_{C}(b+c)^{3}}{3 E I}-\frac{F b^{3}}{3 E I}-\frac{F b^{2}}{2 E I} \cdot c-(b+c) \cdot \theta_{B}=0$

Applying equilibrium law upon Fig.4, and taking moments about point $B$, one reads

$F_{A} a+F_{C}(b+c)-F b=0$

The angle of rotation of cross section $B, \theta_{B}$ can be obtained from solving the equations (5), (6), and (7) as

$$
\theta_{B}=\frac{F a b c(b+2 c)}{6 E I\left(b^{2}+a b+2 b c+a c+c^{2}\right)}
$$

The reaction $F_{C}$ is found by solving eq.(6) as

$$
F_{C}=\frac{F b\left(2 b^{2}+3 b c+2 a b+2 a c\right)}{2(b+c)^{2}(a+b+c)}
$$

The deflection of section $D$ is

$$
w_{D}=\theta_{B} b+\frac{F b^{3}}{3 E I}-\frac{F_{C} b^{3}}{3 E I}-\frac{F_{C} c \cdot b^{2}}{2 E I}=\frac{F b^{2}\left(4 b^{4}+4 b^{3} a+2 b^{3} c-3 b^{2} c^{2}+4 b c^{3}+4 a c^{3}\right)}{12 E I(b+c)^{2}(a+b+c)}
$$

The rotation of section $D$ is

$$
\theta_{D}=\theta_{B}+\frac{F b^{2}}{2 E I}-\frac{F_{C} b^{3}}{2 E I}-\frac{F_{C} c b}{E I}=-\frac{F b c\left(3 b^{3}+4 b^{2} a-6 b c^{2}-4 a c^{2}\right)}{12 E I(b+c)^{2}(a+b+c)}
$$

Example 2. A simple rigid frame $A B C$ supports the uniform distributed load of intensity $q$, as shown in Fig.6. Determine the angle of rotation $\theta_{B}$. Neglect the axial deformations and assume $E I$ is constant.

Solution. The deformation of rigid frame $A B C$ under the uniform distributed load of intensity $q$ is approximately shown in Fig.7.

To use the method of ERB for finding the angle $\theta_{B}$, the rigid frame $A B C$ is divided into two segments $A B$ and $B C$. Since the end $A$ is fixed, assuming that the angle of rotation at $B$ is $\theta_{B}$, and the interactions between two segments $A B$ and $B C$ are $F_{B}$ and $M_{B}$, then the ERBs of rigid frame $A B C$ in Fig. 8 can be built as shown in Fig.8.

By the geometric relation in Fig.8, one reads

$$
\overline{C_{1} C_{2}}=b \cdot \theta_{B}
$$




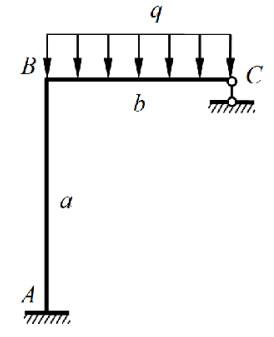

Fig.6 A simple rigid frame supports the distributed load

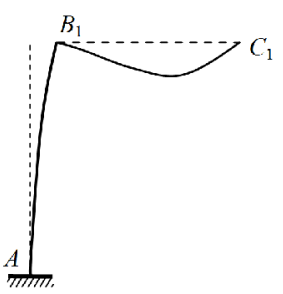

Fig.7 The deformation of rigid frame $A B C$

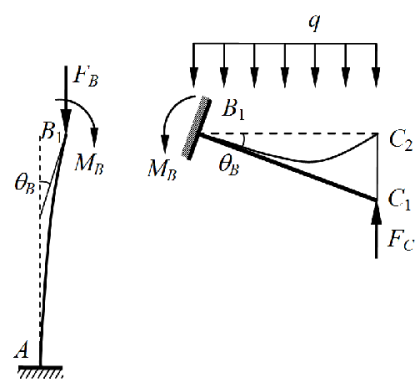

Fig.8 The ERBs $A B_{1}$ and $B_{1} C_{1}$ of frame $A B C$ in Fig.6

Employing the method of superposition and (1), (2) and (3), one reads

$$
\begin{aligned}
& \frac{F_{C} b^{3}}{3 E I}-\frac{q b^{4}}{8 E I}-b \theta_{B}=0 \\
& \frac{M_{B} a}{E I}=\theta_{B}
\end{aligned}
$$

Applying equilibrium law upon the ERB $B_{1} C_{1}$, by taking moments about point $B$, one reads

$$
M_{B}+F_{C} b-\frac{1}{2} q b^{2}=0
$$

Then, $\theta_{B}$ can be obtained from solving the equations (12), (13) and (14) as

$$
\theta_{B}=\frac{q b^{3} a}{8 E I(3 a+b)}
$$

\section{Conclusions}

Illustrative Examples given in this paper reveal that the method of ERB has strong simplicity, validity and applicability for solving deformations of statically indeterminate beams or rigid frames. Method of ERB can give a clear clue to find deflections of beams, and has distinct concepts of mechanics. The concept of ERB give students and researchers a useful tool to investigate the deformations of beams or frames. Examples given in this paper demonstrate that this method is very easy to understand, accept and apply to solve specific problems. Whereas, the method of ERB for statically indeterminate beams or rigid frames, as for statically determinate beams, is only suitable for linear beams or rigid frames with constant cross sections.

\section{References}

1. C. Truesdell, Outline of the History of Flexible or Elastic Bodies to 1788. The Journal of the Acoustical Society of America 32, 1647-56 (1960). doi: 10.1121/1.1907980.

2. Timoshenko, S., "History of strength of materials", McGraw-Hill New York, 1953.

3. James M. Gere, "Mechanics of Materials", fifth ed., Brooks/Cole Publishing Company, Thomson Learning, 2001.

4. R.C.Hibbeler. "Mechanics of Materials", Fifth Edition. Pearson Education, Prentice Hall. 2003.

5. Sun Xunfang, Fang Xiaoshu, Guan Laitai, "Mechanics of Materials ( $\square$ )" (in Chinese), 5ed., Higher Education Press, Beijing, 2009.

6. Zhenchao, SU; Yanxia, XUE. A Novel Method of Equivalent Replacement Beams for Displacement Computation of Euler-Bernoulli beams. PROCEEDINGS OF THE 2017 6TH INTERNATIONAL CONFERENCE ON ENERGY AND ENVIRONMENTAL PROTECTION (ICEEP 2017), Advances in Engineering Research vol: 143, pp: 1315-1318. 